\title{
Identification des risques climatiques de la culture du maïs au Burkina Faso
}

\author{
Benoît SARR *, Luc KAFANDO et Sanoussi ATTA \\ Centre Régional Agrhymet, Département Formation et Recherche, BP 11011, Niamey, Niger. \\ *Auteur correspondant, E-mail: b.sarr@agrhymet.ne, Tel: 00 (227) $20315316 / 20315436$;
}

Fax: 00 (227) 20315435

\section{RESUME}

Au Burkina Faso, le maïs est cultivé dans les zones soudaniennes et soudano-sahéliennes où les conditions pluviométriques sont plus favorables. La culture est sensible aux aléas climatiques liés à la variabilité et aux extrêmes pluviométriques. L'objectif de l'étude est d'identifier les risques climatiques pour la culture du maïs. A partir des données météorologiques journalières, une analyse agroclimatique a été réalisée sur la période 1979-2008 sur six stations synoptiques. A l'aide du logiciel Instat+ v 3.036, la variabilité des précipitations et des paramètres clés de la saison agricole dont les dates de démarrage, de fin, la longueur de la saison, les occurrences de séquences sèches et la satisfaction des besoins en eau ont été analysés en termes de risques d'apparition. Les résultats ont montré que le maïs est confronté à deux risques agroclimatiques majeurs. Il s'agit, dans la zone soudano-sahélienne, des déficits hydriques imputables à des séquences sèches au cours du développement du maïs. Tandis que les excès d'eau liés à des fortes pluies ou des successions d'épisodes secs et d'excès d'eau constituent les risques en zone soudanienne. La période d'installation, le cumul pluviométrique, la longueur de la saison ne semblent pas constituer de risques agroclimatiques majeurs pour le maïs.

(C) 2011 International Formulae Group. All rights reserved.

Mots clés : Maïs pluvial, variabilité pluviométrique, paramètres saison agricole, déficit hydrique, excès d'eau.

\section{INTRODUCTION}

Au Burkina Faso, le maïs est la céréale la plus cultivée après le sorgho et le mil (DGPER, 2010). La zone de culture du maïs représente trois quart de la superficie totale du pays. Depuis les années 2000, les superficies emblavées en maïs sont en constante augmentation. Ces superficies, évaluées à 608 368 hectares en 2008 représentaient 14,5\% des superficies emblavées en céréales. L'émergence des producteurs de semences améliorées et des fermes semencières grâce à l'appui de l'Etat et de ses partenaires techniques et financiers a donné beaucoup d'importance à la production du maïs par la fourniture de la semence de qualité aux producteurs agricoles. Aujourd'hui, le maïs entre dans les habitudes culinaires et surtout devient une matière première pour les brasseries. Il est essentiellement destiné à l'autoconsommation et procure des revenus monétaires variables selon les exploitations. 
Les résidus de maïs constituent également une source importante d'aliments pour les animaux. Enfin d'une culture à vocation alimentaire, le maïs est devenu progressivement une culture de rente avec une plus grande réceptivité aux améliorations techniques (Wey, 1998). Cependant, la culture du maïs est une activité agricole sensible aux aléas du climat et des conditions météorologiques.

La variabilité spatio-temporelle des pluies au Burkina Faso, comme dans toutes les régions soudano-sahéliennes d'Afrique de l'Ouest, a été reconnue comme l'une des plus importantes au monde (IPCC, 2007). A ces contraintes agro-climatiques s'ajoute le contexte socio-économique et politique souvent défavorable qui expliquent pourquoi les productions restent encore faibles (Traoré et al., 2010).Toutefois, dans les agricultures pluviales des régions soudano-sahéliennes, l'influence des conditions climatiques sur les choix techniques et sur les résultats de production apparaît déterminante. Or, au cours des dernières années 70 et 80 , l'Afrique de l'Ouest a connu une forte diminution des précipitations. Une rupture nette des séries pluviométriques est observée autour des années 1968-1972, 1970 étant une année charnière (Le barbé et al., 2002 ; Abdou et al., 2008). La réduction importante des précipitations apparaît sous forme d'épisodes de forts déficits en 1972-73, 1982-84 et 1997 (Nicholson, 2001). Pour le cas des zones soudano-sahéliennes, cette tendance s'est traduite par un glissement des isohyètes de l'ordre de 150 à $200 \mathrm{~km}$ vers le Sud (Diouf et al., 2000). Dans toute la région, le déficit pluviométrique résulte de la diminution du nombre d'événements pluvieux à une augmentation du nombre d'épisodes secs et une baisse de la contribution des mois les plus pluvieux (août et juillet) au cumul pluviométrique (Lebel et al., 2003 ; Gachon et al., 2007). Cette irrégularité des pluies a pour corollaire la variabilité des dates de semis, des dates de fin et la durée de la saison des pluies et une plus grande occurrence des déficits hydriques (Traoré, 2000). Cette variabilité des pluies est souvent conjuguée à des événements extrêmes climatiques qui ont des conséquences désastreuses sur la production agricole et les revenus des populations. Les sécheresses des années 1970 et 1980 et les fortes pluies récurrentes qui ont frappé la zone et ses habitants au cours des décennies 1990 et 2000 constituent un des événements extrêmes qui seraient amplifiés sans doute par le changement climatique. Or, le changement climatique dû à la modification de la teneur en gaz de l'atmosphère indique diverses tendances qui se manifestent déjà au niveau mondial et africain par une hausse des températures (Christensen et al., 2007), une variabilité accrue de la pluviométrie et des caractéristiques des saisons agricoles, une recrudescence de phénomènes extrêmes tels que les sécheresses et inondations (IPCC, 2007). C'est ainsi qu'à partir des années 1990 et 2000, des conditions pluviométriques bien meilleures se sont réinstallées en Afrique soudano-sahélienne (Ali et al., 2008). Ces conditions favorables sont, le plus souvent, associées à des pluies parfois intenses occasionnant des inondations et de nombreux dégâts en plusieurs endroits en Afrique de l'Ouest. En 2007, une des années de pires inondations en Afrique de l'Ouest depuis plus de 30 ans, 17689 ha de cultures ont été inondées au Burkina Faso. Ceci a occasionné des pertes de production agricole de 13500 tonnes (Sarr et Issaka, 2009). De nombreux auteurs (Janicot et al., 2001) ont mis en évidence le lien entre les anomalies de température de surface dans l'atlantique Nord et le Sud, le phénomène planétaire El Niño et la sécheresse au Sahel. Des épisodes secs réguliers intervenant au cours du cycle de développement des cultures occasionnent ainsi une baisse des rendements des 
principales cultures alimentaires (Sivakumar, 1988). Tadross et al. (2009) ont montré que les années d'apparition d'El Niño sont associées à des modifications de la durée des séquences sèches durant la saison pluvieuse dans des pays comme le Malawi, la Mozambique, la Zambie et le Zimbabwe. C'est ainsi que les séquences sèches en année El Niño ont des durées moyennes de 14 jours contre 10 jours en année La Niña (Stern et Cooper, 2011). Enfin, le raccourcissement de la période de croissance végétative consécutif à un arrêt brutal des pluies avant la fin de la saison des pluies constitue une des contraintes agroclimatiques majeures de la production agricole pluviale (Araya et Stroosnijder, 2011).

Ces anomalies pluviométriques peuvent se traduire par des baisses de la production en grain de plus de $60 \%$ (Watson et al., 1998) et entravent par conséquent le développement social et économique.

Il s'agit là de tendances très lourdes pour l'agriculture et les économies locales essentiellement basées sur l'exploitation des ressources naturelles fortement sensibles au climat et à ses variations.

Le maïs est une plante exigeante en eau. En outre, elle présente une plus grande sensibilité à la sécheresse (Sarr et al., 1999) par rapport aux autres céréales sèches comme le mil et le sorgho. Les besoins en eau du maïs augmentent avec la longueur du cycle et le type de climat. Suivant les climats, il lui faut entre $400 \mathrm{~mm}$ et $800 \mathrm{~mm}$ de pluie bien répartie pour boucler son cycle avec une somme de température de 1500 à 1800 degré jours (Doorenbos et Kassam, 1980). Cependant l'excès d'eau peut provoquer la pourriture des racines et l'asphyxie des plants de maïs. Plus que le cumul pluviométrique total enregistré sur l'ensemble du cycle, le rendement du maïs dépend essentiellement de la disponibilité en ressources hydriques pendant les phases critiques de son développement (Robelin, 1963).

Pour faire face aux risques climatiques, il est possible d'agir en prévention de l'aléa climatique par l'exploitation des techniques agricoles telles que l'irrigation, l'utilisation de variétés adaptées, le respect du calendrier cultural, l'utilisation des techniques de conservation des eaux et des sols (Chetaille et Lagandé, 2010). Une bonne connaissance des risques climatiques majeurs de la culture s'impose.

L'objectif de l'étude est d'identifier les risques climatiques majeurs qui peuvent causer des dégâts et des pertes de récolte à la culture du maïs au Burkina Faso.

Dans le contexte climatique du Burkina Faso, plusieurs hypothèses de risques climatiques de la culture du maïs pluvial ont été émises: (i) le cumul pluviométrique saisonnier est inférieur aux besoins en eau totaux du maïs; (ii) les besoins en eau du maïs pour une phase donnée ne sont pas satisfaits par le cumul pluviométrique durant cette phase ; (iii) la variabilité de la date de début de la saison des pluies ne permet pas aux producteurs de respecter la date favorable de semis du maïs pour la zone; (iv) la longueur de la saison des pluies est plus courte que la longueur du cycle du maïs ; (v) des séquences sèches dommageables à la productivité du maïs apparaissent au cours de son cycle de développement; (vi) des cas d'excès d'eau consécutifs à des pluies extrêmes maximales surviennent au cours du cycle de développement du maïs.

\section{MATERIEL ET METHODES}

L'étude couvre la zone maïsicole du Burkina Faso qui comprend la zone soudanienne avec un cumul pluviométrique moyen $\geq 800 \mathrm{~mm} \mathrm{an}^{-1}$ et la zone soudanosahélienne à cumul pluviométrique compris entre 600 et $800 \mathrm{~mm} \mathrm{an}^{-1}$ (Figure 1). 


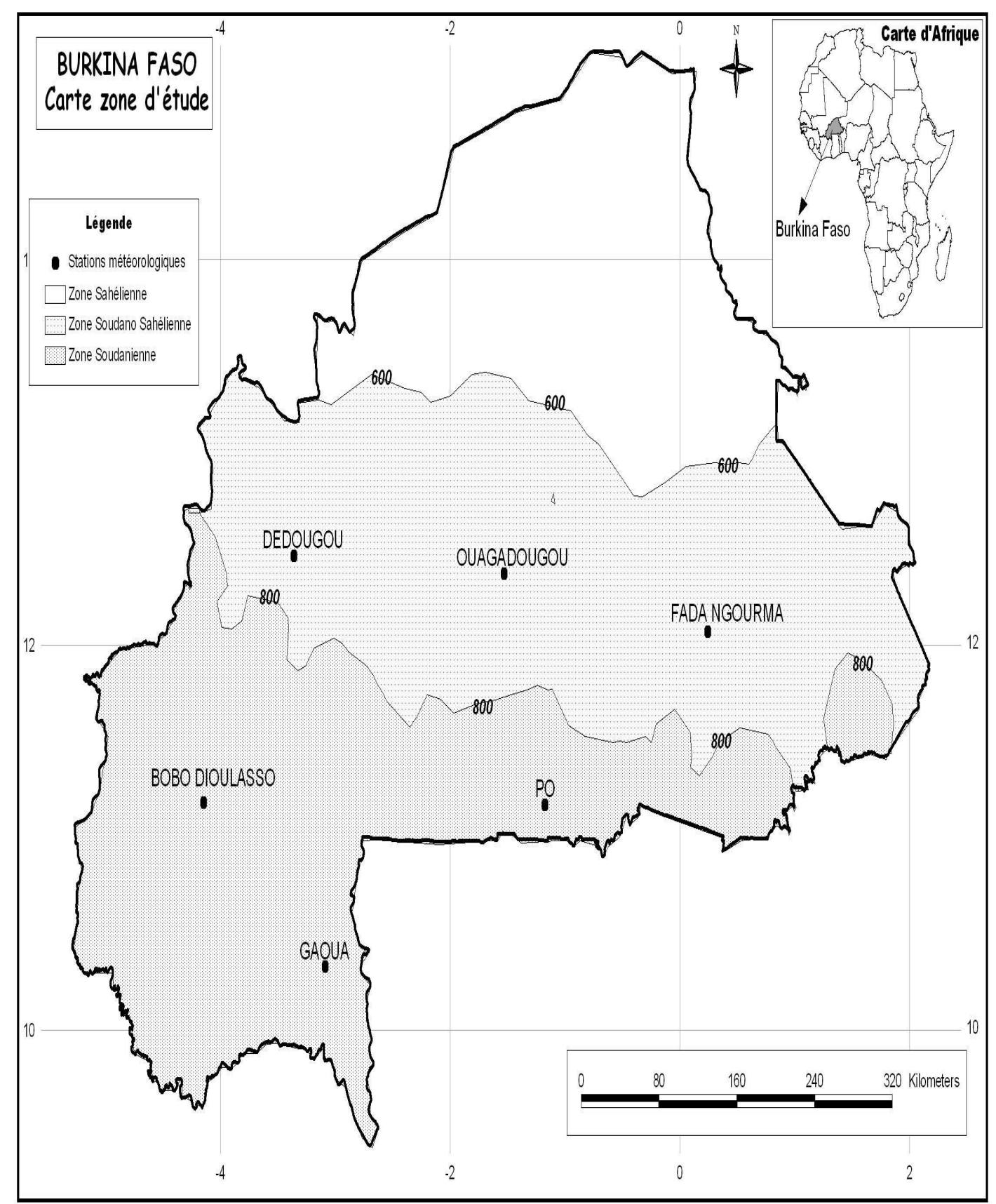

Figure 1 : Les zones agro climatiques du Burkina Faso et la zone de culture de maïs au Sud de l'isohyète $600 \mathrm{~mm}$. 


\section{Données météorologiques}

Les données pluviométriques journalières de 1979 à 2008 et les valeurs moyennes décadaires de l'évapotranspiration potentielle (ETP) Penman de 1984 à 2008 de 3 stations météorologiques représentatives de la zone soudanienne et 3 de la zone soudanosahélienne, (Tableau 1) ont été utilisées. Les données ont été recueillies auprès de la Direction Nationale de la Météorologie du Burkina Faso.

La normale 1979 -2008 est la période où toutes les stations avaient des données de pluies journalières complètes. En outre, cette période dite «normale» a l'avantage de rassembler les années sèches de la période. 1980-1990 et les décennies 1990 et 2000 marquées par une alternance d'années sèches et humides.

\section{Matériel végétal}

$\mathrm{Au}$ Burkina Faso, l'Institut de l'Environnement et de Recherches Agricoles a mis au point des variétés améliorées de maïs en fonction des zones agroécologiques. Les variétés précoces ( 85 à 94 jours) et les variétés extra-précoces (75 à 84 jours) sont recommandées pour les zones à pluviométrie inférieure à $900 \mathrm{~mm}$. Quant aux variétés à cycle intermédiaire de l'ordre de 95 à 110 jours, elles sont recommandées pour la zone soudanienne (Wey, 1998).

\section{Méthodologies pour l'analyse des risques climatiques}

Afin de vérifier les hypothèses émises, diverses analyses agroclimatiques sur la variabilité de la pluviométrie et des paramètres clés de la saison des pluies ont été déterminées à l'aide du logiciel Instat+v.3.036 (Stern et al., 2006).

Dates de début, de fin et de la longueur de la saison agricole

Dans la littérature scientifique, plusieurs critères basés sur des seuils de pluies
(Stern, 1981 ; Traoré et al., 2000 ; Omotosho et al., 2000; Camberlin et Diop, 2003) sur le rapport entre la pluviométrie et l'évapotranspiration potentielle (Cochemé et Franquin, 1967) et enfin sur le bilan hydrique (Samba, 1998). Dans cette étude, les dates de démarrage de la saison ont été déterminées en utilisant le critère le premier jour, à partir du premier avril, lorsqu'on enregistre plus de 20 mm de pluie en 1 ou 2 jours successifs mais sans épisode sec de plus de 7 jours dans les 30 jours qui suivent le semis (Somé et Sivakumar, 1994). Les dates de fin de la saison des pluies ont été déterminées en considérant à partir du début septembre le moment où le maïs a épuisé la réserve hydrique du sol. Les valeurs des réserves utiles en eau du sol utilisées pour chaque station sont celles proposées par Bunasols (2003). La longueur de la saison est exprimée par la différence entre la date de fin et la date de début de la saison des pluies.

\section{Pluviométrie et séquences sèches}

Les cumuls pluviométriques saisonniers moyens ont été calculés puis confrontés avec les besoins en eau totaux du maïs. Les pluies maximales journalières cumulées en 3 jours consécutifs ont été déterminées ainsi que leur durée de retour.

Dans cette étude, on a considéré comme séquence sèche un nombre de jours consécutifs sans pluie pour une période donnée. Un jour où le cumul pluviométrique journalier est inférieur à $0,1 \mathrm{~mm}$ a été considéré comme jour sans pluie. Les séquences sèches de $>7$ et 10 jours durant les 30 jours qui suivent le semis et celles $>5,7$ et 10 jours pendant la floraison (40 - 65 jours après le semis) du maïs 90 jours ont été calculées. Leur apparition constitue des risques majeurs pour la culture du maïs. En outre, les dates favorables au semis ont été déterminées. Il s'agit, selon Stern et al. (2006), de la date à partir de laquelle le niveau 
de probabilité d'apparition de séquences sèches $>10$ jours est faible $(\leq 20 \%)$.

\section{Indice I de satisfaction des besoins en eau du maïs}

Frère et Popov (1987) ont mis au point pour le compte de la FAO, une méthode d'estimation de l'indice I de satisfaction des besoins en eau des cultures dans les pays où l'eau constitue un facteur limitant de l'agriculture pluviale. L'indice exprime le degré auquel ont été satisfaits les besoins en eau cumulés de la plante à un moment donné ou pour la totalité du cycle végétatif (Tableau 2). Il permet également de suivre les déficits hydriques tout au long de la période de croissance, en tenant compte des stades phénologiques et des périodes dans lesquelles la disponibilité d'eau est la plus critique pour le développement de la culture. Le bilan hydrique est basé sur un principe relativement simple.

Au début de la saison des pluies, on attribue la valeur 100 à I tout en présumant qu'au moment du semis, la réserve d'eau dans le sol est supérieure aux besoins en eau des plantes. Les valeurs de I diminuent dès que survient un stress hydrique.

En cas de déficit (Di), l'indice est réduit du pourcentage de ce déficit par rapport aux besoins totaux en eau pour la saison TMR.

$\mathrm{Si}(\mathrm{Ei} / \mathrm{Di})<0$ alors $\mathrm{Ii}=\mathrm{I}_{\mathrm{i}-1}-(\mathrm{Ei} / \mathrm{Di} * 100) /$ $\mathrm{TMR}$, sinon $\mathrm{Ii}=\mathrm{I}_{\mathrm{i}-1}$.

$\mathrm{TMR}=\sum_{I=1}^{n} K c_{i} * E_{i}$

Kci : coefficient cultural de la plante à un stade phénologique et une décade i donnés (Dancette, 1983)

ETP $i$ : évapotranspiration potentielle de la décade i ; Ei : excédent d'eau dans le sol.

En cas de surplus d'eau dans le sol supérieur à $100 \mathrm{~mm}$, considéré comme excédent d'eau nuisible à la plante, l'indice sera réduit de 3 unités. $\mathrm{Si}(\mathrm{Ei} / \mathrm{Di})>100, \mathrm{Ii}=$ $\mathrm{I}_{\mathrm{i}-1}-3$
A maturité de la plante, l'index I de satisfaction des besoins en eau reflétera l'ensemble cumulé des déficits d'eau subis décade après décade, par la plante durant le cycle.

Une valeur élevée de I (99 à 100) traduit des conditions d'alimentation hydrique bonnes à très bonnes et des valeurs faibles de 59 à 60, des conditions mauvaises voire médiocres. Des valeurs de $\mathrm{I}<50$ correspondent à un échec total de la culture. Les valeurs saisonnières de l'indice de satisfaction des besoins en eau des cultures ont été calculées. Les causes des variations de l'indice I à savoir le déficit, l'excès, ou à la fois déficit et l'excès d'eau et leur probabilité d'apparition ont été analysées.

\section{Analyse statistique des variables agrométéorologiques}

A l'aide de Instat + v 3.36, des statistiques descriptives (cumul, moyenne interannuelle, écart type, coefficient de variation) ont été appliquées aux variables agroclimatiques. Des analyses fréquentielles ont été réalisées pour les niveaux de probabilités 20, 50 et $80 \%$. La durée de retour de 2,5 et 10 ans des pluies maximales a été calculée comme suit :

Durée de retour $(a n s)=\frac{100}{100-(\mathrm{Fx})}$

avec Fx: fréquence cumulée après ajustement à une loi de distribution de type Gumbel (Djerboua et al., 2004). L'analyse des probabilités d'apparition d'événements agroclimatiques (occurrence de séquences sèches, date de démarrage de saison tardive, fin de saison précoce, hivernage court, pluies maximales journalières, déficit ou excès d'eau) dommageables aux cultures a permis d'identifier les risques climatiques de la culture du maïs. Pour les paramètres de la saison agricole, les niveaux de probabilités 20 , 50 et $80 \%$ correspondent respectivement aux dates d'installation et de fin saison précoce, normale et tardive des pluies. Quant à la 
longueur de la saison, ces probabilités correspondent respectivement à des saisons courtes, normales et longues.

\section{RESULTATS}

\section{Pluviométrie et séquences sèches}

Le cumul pluviométrique moyen annuel de la période 1979-2008 est compris entre $710 \mathrm{~mm}$ à Ouagadougou et $1045 \mathrm{~mm}$ à Gaoua. Huit années sur dix (ou dans $80 \%$ des années), des cumuls pluviométriques sont supérieurs ou égaux compris entre $620 \mathrm{~mm}$ à Ouagadougou et $920 \mathrm{~mm}$ à Gaoua (tableau 2) sont enregistrés. Autrement dit, ce n'est que dans $20 \%$ des années que l'on enregistre des totaux annuels inférieurs ou égaux compris entre $620 \mathrm{~mm}$ à Ouagadougou et $920 \mathrm{~mm}$ à Gaoua.

\section{Analyse des séquences sèches > 7 et 10 jours}

Séquences sèches > 7 et >10 jours après le semis

Dans la zone soudanienne, entre mi et fin avril, les probabilités de séquences sèches $>7$ jours après le semis sont élevées avec un minimum de $40 \%$ à Bobo pour un maximum de $87 \%$ à Po. Elles décroissent rapidement pour atteindre le seuil de $20 \%$ vers le 20 Mai pour la station de Bobo et Po. La décroissance est moins rapide à Gaoua (Figure 2).

Dans la zone soudano-sahélienne, les probabilités d'apparition de séquences sèches $>7$ jours après le semis restent élevées entre le 16 et le 30 Avril comparativement à la zone soudanienne. Elles varient entre $70 \%$ à Fada, à $97 \%$ à Ouagadougou. Elles diminuent linéairement durant tout le mois de Mai mais restent toujours supérieures à $30 \%$ jusqu' au 5 Juin.

Les probabilités d'apparition de séquences sèches > 10 jours après le semis dans la zone soudanienne ne sont élevées qu'entre le 16 Avril et le 7 Mai à la station de Po (60-30\%). Les probabilités deviennent $\leq$
$20 \%$ à Gaoua le 22 Avril, à Bobo le 29 Avril et à Po le 10 Mai (Figure 3). En zone soudano-sahélienne, elles restent encore élevées soit 60 - $82 \%$ en mi avril puis chutent rapidement après cette date. Vers le 10 juin elles sont de l'ordre de $20 \%$ pour toutes les stations de la zone.

\section{Séquences sèches > 5 et $>7$ jours pendant la floraison du maïs}

La période de floraison du maïs de 90 jours semé entre le $1^{\text {er }}$ et le 21 Mai dans la zone soudanienne, coïncide avec des probabilités d'apparition de séquences sèches $>5$ jours élevées dans les stations de Gaoua et de Po. Ces probabilités tournent autour de $50 \%$ avec des valeurs maximales de $60 \%$ à Po pour les semis de début Mai. Du 22 Mai au 10 Juin, elles diminuent mais restent supérieures à $30 \%$. Par contre à Bobo, les probabilités d'apparition de séquences sèches $>5$ jours au cours de la floraison du maïs sont faibles $(\leq$ $20 \%$ ) pour les différentes dates de semis exceptées les semis précoces de début Mai dont les probabilités d'apparition des séquences sèches sont de 30\% (Tableau 3).

Dans la zone soudano-sahélienne, la période de floraison pour des semis effectués en mai coïncide avec des probabilités élevées d'apparition de séquences sèches $>5$ jours à Dédougou et à Ouagadougou. Elles oscillent entre 38 et $79 \%$ à Dédougou et entre 67 et $33 \%$ à Ouagadougou. A Fada, ces niveaux de probabilités sont plus faibles pour les semis compris entre le 10 Mai et le 10 Juin $(\leq 30 \%)$. Excepté la station de Bobo, les probabilités d'apparition de séquences sèches diminuent de moitié entre les premiers semis du mois de Mai et ceux de Juin (Tableau 4).

Dans la zone soudano-sahélienne, les probabilités d'apparition de séquences sèches $>7$ jours pendant la floraison sont nulles à faibles $(\leq 20)$ aux stations de Ouagadougou et de Fada pour la plage de semis du $1^{\text {er }}$ Mai au 10 Juin. Quant à la station de Dédougou les niveaux de probabilités comprises entre 17- 
$34 \%$ sont plus élevés pour les semis réalisés entre le $1^{\text {er }}$ et le 16 Mai (Tableau 4).

\section{Pluies maximales journalières}

En zone soudanienne, les pluies maximales cumulées en 3 jours consécutifs décennales varient entre $140 \mathrm{~mm}$ à Po à 123 $\mathrm{mm}$ à Bobo. Dans la zone soudanosahélienne, elles sont comprises entre $113 \mathrm{~mm}$ à Fada et $107 \mathrm{~mm}$ à Ouagadougou. Les valeurs des pluies quinquennales oscillent entre 119 et $108 \mathrm{~mm}$ dans la zone soudanienne et entre 88 et $100 \mathrm{~mm}$ dans la zone soudano-sahélienne. Enfin, une année sur 2, ces pluies restent encore élevées et sont comprises entre $88 \mathrm{~mm}$ dans la zone soudanienne et de $78 \mathrm{~mm}$ dans la zone soudano-sahélienne.

\section{Dates de démarrage, de fin et longueur de la saison agricole}

La date d'installation de la saison des pluies présente une grande variabilité spatiotemporelle dans la zone maïsicole du Burkina. L'installation a lieu en moyenne entre mi mai au Sud du pays à mi juin au Nord de la zone de culture du maïs. Dans $80 \%$ des années, l'installation de la saison des pluies a lieu avant mi juin en zone soudanienne. Tandis que dans la zone soudano-sahélienne, elle a lieu avant fin juin - début juillet (Tableau 6).

En moyenne, la saison des pluies prend fin au cours de la première décade du mois d'octobre en zone soudano-sahélienne. En zone soudanienne, l'hivernage prend fin entre mi-octobre et le 24 octobre (non montré). L'analyse montre également que dans $80 \%$ des années, l'hivernage prend fin avant fin octobre dans la zone soudanienne et avant une date comprise entre le 16 et le 21 octobre dans la zone soudano-sahélienne (non montré).

La longueur moyenne varie en moyenne de 110 à 120 jours en zone soudanosahélienne et de 140 à 165 jours en zone soudanienne (Tableau 7). Dans 20\% des années, la longueur de la saison est $\leq$ à des valeurs comprises entre 123 et 143 jours, autrement dit dans $80 \%$ des cas, elle est $\geq$ à ces valeurs dans la zone soudanienne. En zone soudano-sahélienne, dans $80 \%$ des années, la longueur de l'hivernage est au moins équivalente à 90 jours.

\section{Indice I de satisfaction des besoins en eau}

Dans la zone d'étude dans 43 à $57 \%$ des années la valeur de l'indice I est de $100 \%$ notamment en zone soudanienne (Tableau 8). Dans $30 \%$ des années, on enregistre sauf à Ouagadougou partout des valeurs de I comprises entre 95 et $99 \%$ matérialisant des bonnes conditions d'alimentation hydrique du maïs. Une année sur cinq (20\%), le maïs est en conditions d'alimentation en eau moyenne (80 et 94\%). Cependant, à Ouagadougou cette situation intervient à peu près dans $40 \%$ des années. Les cas de rendements médiocres dus à des valeurs de I comprises entre 60-79\% sont peu fréquents soit moins de $10 \%$ des années.

Il est a noté que les cas de baisse de l'indice I ( < 100) quoique plus élevés en zone soudano-sahélienne soit plus de 50 à $60 \%$ des années sont aussi importants en zone soudanienne (Tableau 8). Toutefois, les causes de la baisse de I sont souvent différentes entre les 2 zones. En zone soudanienne, la baisse de I est le plus souvent imputable à des surplus d'eau, soit 27 à 37\% des années (Tableau 9). Des déficits hydriques ou des surplus d'eau associés à des déficits hydriques peuvent l'expliquer également dans $10 \%$ des années environ. En revanche, en zone soudano sahélienne, la baisse de I est préférentiellement due aux déficits hydriques. Le nombre de fois où I baisse sur les 30 ans est de $57 \%$ à Ouagadougou, $53 \%$ à Dédougou et $37 \%$ à Fada N'Gourma. On y rencontre aussi des cas moins fréquents $(<10 \%)$ de déficits associés à des surplus. 
Tableau 1 : Listes des stations météorologiques utilisées dans l'étude.

\begin{tabular}{llll}
\hline Stations & Zones agroécologiques & Longitude & Latitude N \\
\hline Fada Ngourma & Soudano-sahélienne & $0^{\circ} 21 \mathrm{E}$ & $12^{\circ} 4$ \\
Dédougou & Soudano-sahélienne & $3^{\circ} 29 \mathrm{O}$ & $12^{\circ} 28$ \\
Ouagadougou & Soudano-sahélienne & $1^{\circ} 31 \mathrm{O}$ & $12^{\circ} 21$ \\
Bobo Dioulasso & Soudanienne & $4^{\circ} 18 \mathrm{O}$ & $11^{\circ} 10$ \\
Gaoua & Soudanienne & $3^{\circ} 11 \mathrm{O}$ & $10^{\circ} 20$ \\
Po & Soudanienne & $1^{\circ} 9 \mathrm{O}$ & $11^{\circ} 10$ \\
\hline
\end{tabular}

Tableau 2: Variabilité des cumuls pluviométriques annuels (en mm) 1979-2008 des 6 stations météorologiques étudiées.

\begin{tabular}{|c|c|c|c|c|c|c|}
\hline \multirow[b]{2}{*}{ Statistique } & \multicolumn{6}{|c|}{ Stations météorologiques } \\
\hline & Bobo & Gaoua & Po & Dédougou & Fada & Ouaga \\
\hline & \multicolumn{3}{|c|}{ Zone soudanienne } & \multicolumn{3}{|c|}{ Zone soudano-sahélienne } \\
\hline Minimum & 778 & 714 & 546 & 396 & 568 & 571 \\
\hline Maximum & 1331 & 1436 & 1290 & 1132 & 1073 & 900 \\
\hline Moyenne & 996 & 1046 & 960 & 761 & 804 & 708 \\
\hline Ecart type $(\sigma)$ & 151 & 153 & 191 & 140 & 137 & 85 \\
\hline Percentile $20 \%$ & 846 & 919 & 786 & 669 & 665 & 622 \\
\hline Percentile $50 \%$ & 959 & 1042 & 919 & 739 & 788 & 700 \\
\hline Percentile $80 \%$ & 1149 & 1173 & 1151 & 891 & 929 & 792 \\
\hline
\end{tabular}

Tableau 3: Probabilités de séquences sèches $>5$ jours (chiffres à gauche) et $>7$ jours (chiffres à droite) pendant la floraison du maïs dans la zone soudanienne pour une variété de 90 jours.

\begin{tabular}{|c|c|c|c|c|}
\hline \multirow[t]{2}{*}{ Date de semis } & \multirow[t]{2}{*}{ Période de floraison } & \multicolumn{3}{|c|}{$\begin{array}{l}\text { Probabilités }(\%) \text { d'apparition de } \\
\text { séquences sèches }\end{array}$} \\
\hline & & Bobo & Gaoua & Po \\
\hline & & \multicolumn{3}{|c|}{$>5->7$ jours } \\
\hline $1^{\mathrm{er}}$ Mai & 9 Juin-4 Juillet & $30-7$ & $57-30$ & $60-23$ \\
\hline $6 \mathrm{Mai}$ & 14 Juin - 9 Juillet & $30-3$ & $53-23$ & $50-17$ \\
\hline 11 Mai & 19 Juin-14 Juillet & $20-0$ & $53-23$ & $47-20$ \\
\hline $16 \mathrm{Mai}$ & 24 Juin- 19 Juillet & $20-3$ & $47-17$ & $50-17$ \\
\hline $21 \mathrm{Mai}$ & 29 Juin- 24 Juillet & $20-7$ & $50-10$ & $47-10$ \\
\hline 26 Mai & 4 Juillet -29 Juillet & $17--7$ & $37-10$ & $40-10$ \\
\hline 31 Mai & 9 Juillet - 3 Août & $20-7$ & $37-10$ & $30-7$ \\
\hline 5 Juin & 14 Juillet -8 Août & $20-0$ & $33-7$ & $33-3$ \\
\hline 10 Juin & 19 Juillet - 13 Août & $17-3$ & $27-7$ & $33-3$ \\
\hline
\end{tabular}


Tableau 4: Probabilités de séquences sèches $>5$ et $>7$ jours pendant la floraison du maïs en zone soudano-sahélienne pour une variété de 90 jours.

\begin{tabular}{lllcc}
\hline Date de semis & Période de & \multicolumn{3}{l}{ Probabilités (\%) d'apparition de séquences sèches } \\
\cline { 3 - 5 } & floraison & Dédougou & Fada & Ouaga \\
\hline \multirow{2}{*}{ 1 $^{\text {er }}$ Mai } & 9 Juin-4 Juillet & $79-34$ & $45->7$ jours & $67-20$ \\
6 Mai & 14 Juin - 9 Juillet & $59-24$ & $37-13$ & $63-13$ \\
11 Mai & 19 Juin-14 Juillet & $48-17$ & $30-7$ & $47-7$ \\
16 Mai & 24 Juin- 19 Juillet & $52-21$ & $30-3$ & $40-3$ \\
21 Mai & 29 Juin- 24 Juillet & $48-14$ & $30-3$ & $37-3$ \\
26 Mai & 4 Juillet - 29 Juillet & $38-17$ & $26-7$ & $33-3$ \\
31 Mai & 9 Juillet - 3 Août & $39-11$ & $23-3$ & $33-3$ \\
5 Juin & 14 Juillet - 8 Août & $21-0$ & $26-7$ & $20-0$ \\
10 Juin & 19 Juillet - 13 Août & $14-0$ & $23-7$ & $10-0$ \\
\hline
\end{tabular}

Tableau 5: Pluies maximales journalières cumulées en 3 jours consécutifs pour une durée de retour de 10,5 et 2 ans (1979-2008).

\begin{tabular}{lcccccc}
\hline \multirow{2}{*}{$\begin{array}{l}\text { Durée } \\
\text { retour (ans) }\end{array}$} & de & \multicolumn{6}{c}{ Stations météorologiques } \\
\cline { 2 - 7 } & Bobo & Gaoua & Po & Dédougou & Fada & Ouaga \\
\hline 10 & 123 & 135 & 140 & 96 & 113 & 107 \\
5 & 108 & 116 & 119 & 88 & 100 & 96 \\
2 & 85 & 89 & 89 & 75 & 81 & 79 \\
\hline
\end{tabular}

Tableau 6: Variabilité des dates de démarrage de la saison des pluies (1979-2008) dans la zone d'étude.

\begin{tabular}{lllllll}
\hline Stations & Bobo & Gaoua & \multicolumn{1}{c}{ Po } & \multicolumn{1}{c}{ Dédougou } & Fada & Ouaga \\
\hline Minimum & 10 avr & 02 avr & 14 avr & 18 avr & 03 mai & 03 mai \\
Maximum & 11 juil & 23 juin & 13 juil & 25 juil & 18 juil & 26 juil \\
Moyenne & 19 mai & 13 mai & 30 mai & 06 juin & 10 juin & 17 juin \\
$\sigma$ & 23 & 23 & 17 & 26 & 18 & 20 \\
Percentile 20\% & 28 avril & 23 avril & 19 mai & 17 mai & 26 mai & 27 mai \\
Percentile $50 \%$ & 14 mai & 17 mai & 27 mai & 6 juin & 10 juin & 20 juin \\
Percentile $80 \%$ & 12 juin & 31 mai & 12 juin & 01 juil & 28 juin & 02 juil \\
\hline
\end{tabular}


Tableau 7: Variabilité de la longueur de la saison des pluies (1979 - 2008) dans la zone d'étude.

\begin{tabular}{lcccccc}
\hline Stations & Bobo & Gaoua & P0 & Dédougou & Fada & Ouaga \\
\hline Minimum & 109 & 106 & 75 & 75 & 80 & 58 \\
Maximum & 207 & 205 & 200 & 165 & 161 & 161 \\
Moyenne & 158 & 164 & 138 & 121 & 120 & 111 \\
$\sigma$ & 26 & 25 & 23 & 24 & 23 & 23 \\
Percentile 20\% & 138 & 143 & 123 & 98 & 93 & 90 \\
Percentile 50\% & 158 & 163 & 138 & 120 & 125 & 109 \\
Percentile 80\% & 183 & 192 & 154 & 144 & 141 & 135 \\
\hline
\end{tabular}

Tableau 8: Fréquence $(\%)$ du nombre d'années où l'indice I est $\geq$ à une valeur seuil comprise entre $100 \%$ et $60-79 \%$ au cours de la période $1979-2008$.

\begin{tabular}{lcccccc}
\hline \multicolumn{6}{c}{ Stations météorologiques } \\
\hline & $\begin{array}{c}\text { Bobo } \\
\text { Zone soudanienne }\end{array}$ & $\begin{array}{c}\text { Gaoua } \\
\text { Indice I(\%) }\end{array}$ & \multicolumn{5}{c}{$\begin{array}{c}\text { Po } \\
\text { Fódougou } \\
\text { Zone soudano sahélienne }\end{array}$} \\
100 & 43 & 57 & 47 & 47 & 47 & 30 \\
$95-99$ & 37 & 27 & 27 & 33 & 33 & 20 \\
$80-94$ & 20 & 17 & 20 & 13 & 13 & 37 \\
$60-79$ & - & - & 7 & 7 & 7 & 13 \\
\hline
\end{tabular}

Tableau 9: Causes et fréquence de la baisse de l'indice I de 1979-2008.

\begin{tabular}{lccccc}
\hline & \multicolumn{2}{c}{$\begin{array}{l}\text { Nombre(\%) } \\
\text { des cas } \mathbf{I}<\end{array}$} & \multicolumn{2}{l}{$\begin{array}{l}\text { Causes et fréquence (\%) de la baisse } \\
\text { de l'indice I }\end{array}$} \\
\cline { 3 - 6 } & $\mathbf{1 0 0}$ & $\begin{array}{l}\text { Surplus } \\
100 \mathrm{~mm}\end{array}$ & $\begin{array}{l}\text { Déficits } \\
\text { hydriques }\end{array}$ & $\begin{array}{c}\text { Surplus et } \\
\text { déficits }\end{array}$ \\
\hline Bobo Dioulasso & 57 & 37 & 7 & 13 \\
Gaoua & 43 & 27 & 10 & 7 \\
Po & 53 & 37 & 10 & 7 \\
Dédougou & 67 & 10 & 53 & 3 \\
Fada Ngourma & 53 & 10 & 37 & 7 \\
Ouagadougou & 70 & 7 & 57 & 7 \\
\hline
\end{tabular}




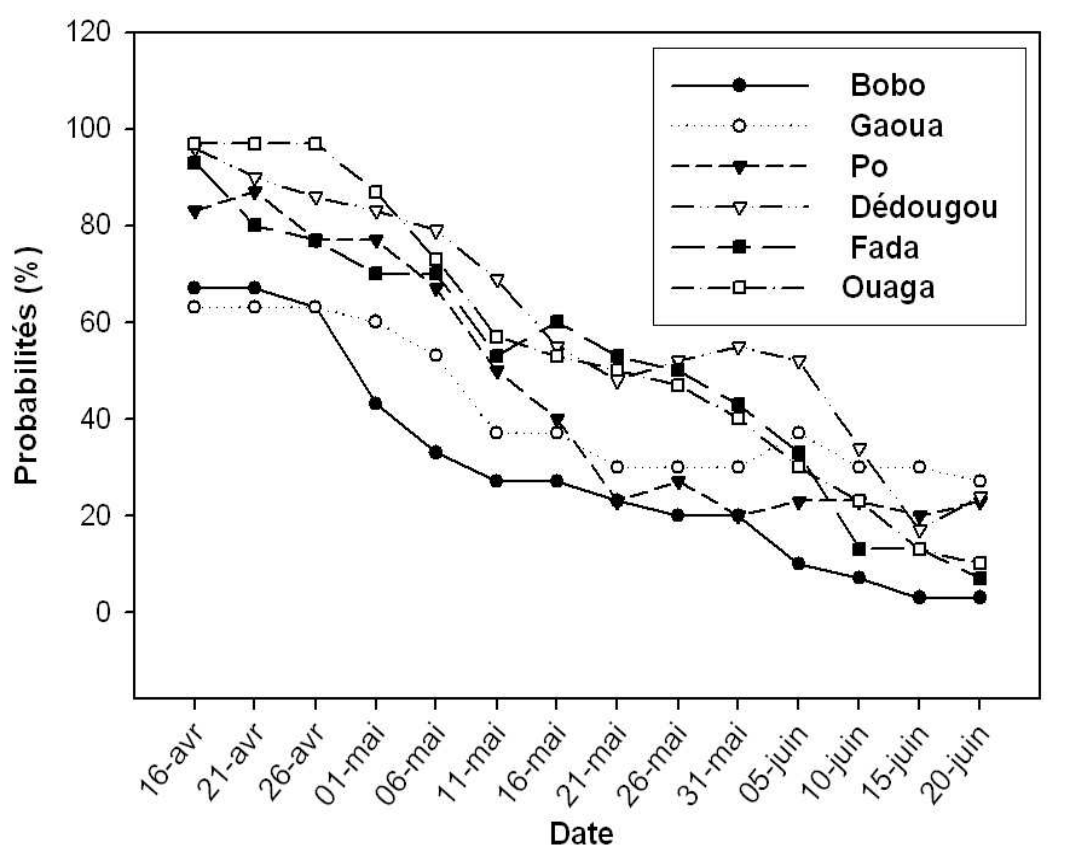

Figure 2 : Probabilités de séquences sèches $>7$ jours, 30 jours après le semis dans la zone soudano-sahélienne et soudanienne.

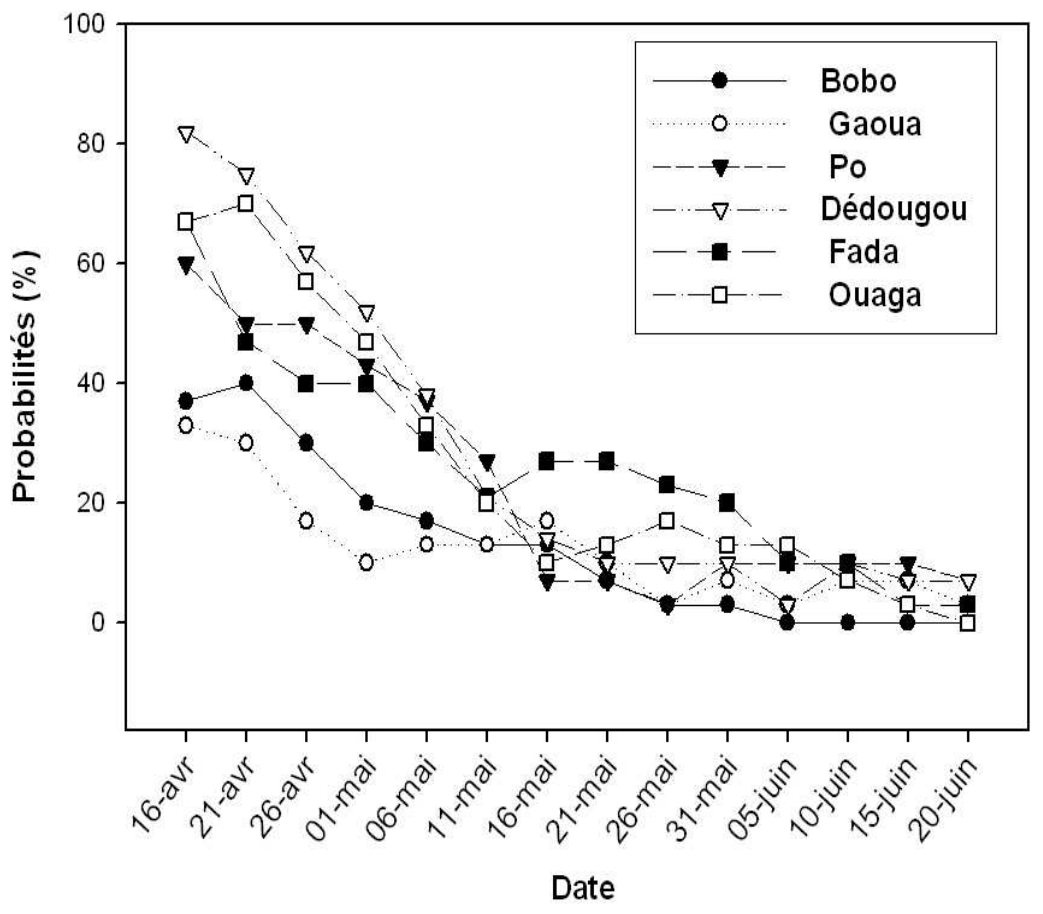

Figure 3 : Probabilités de séquences sèches > 10 jours, 30 jours après le semis dans la zone soudano-sahélienne et soudanienne. 


\section{DISCUSSION}

L'analyse du cumul pluviométrique annuel de la série 1979-2008 a montré que dans 80 des années, le cumul pluviométrique est toujours $\geq 620 \mathrm{~mm}$ en zone soudano sahélienne et peut même atteindre des valeurs de plus de $900 \mathrm{~mm}$ en zone soudanienne. Doorenbos et Kassam (1980) avaient montré que les besoins en eau totaux du maïs varient selon les climats entre 500 et $800 \mathrm{~mm}$ de pluie. Au Burkina Faso, ils se situent entre $415 \mathrm{~mm}$ dans la zone soudanienne et $544 \mathrm{~mm}$ dans la zone sahélienne (Barry, 2007). Dans la zone d'étude, les besoins en eau du maïs pour tout son cycle seront satisfaits chaque année par la pluviométrie totale. La satisfaction des besoins en eau totaux du maïs en considérant les quantités de pluies totales, ne constitue donc pas un risque pour la culture du maïs dans les zones soudanienne et soudanosahélienne du Burkina Faso. Une étude récente de Kambire et al. (2010) au Burkina Faso a montré que le rendement en grain n'est pas affecté par la pluviométrie au cours des deux premiers mois du cycle de développement du maïs. C'est plutôt la pluviométrie du trosième mois après le semis et l'applicatiuon de la fertilisation azotée qui explique la variabilité des rendements.

L'analyse de la longueur de la saison a montré que $80 \%$ des années elle est $\geq 90$ à 100 jours environ en zone soudano -sahélienne. Cette longueur peut atteindre voire dépasser 140 jours en zone soudanienne. La longueur de la saison des pluies est suffisante dans plus de $80 \%$ des années pour que les cultures de maïs de 100 jours dans la zone soudanienne et de 90 jours dans la zone soudano-sahélienne bouclent normalement leur cycle.

Les résultats des dates de démarrage de campagne montrent que la saison des pluies s'installe le plus souvent avant le 30 mai dans la zone soudanienne et avant début juin dans la zone soudano-sahélienne. Les valeurs relativement élevées des écarts type des dates de démarrage par rapport aux dates de fins témoignent d'une plus grande variabilité interannuelle de l'installation de l'hivernage.
Il a été, en effet noté, qu'au cours des dernières années, les débuts et fins des saisons pluvieuses sont devenus de moins en moins prévisibles pour les paysans (Sivakumar, 1988 ; Diop, 1996 ; Diouf et al., 2000).

L'analyse de séquences sèches en début de cycle montre que le maïs peut être semé à partir de Mai dans la zone soudanienne et à partir de la première décade de Juin dans la zone soudano-sahélienne. Toutefois l'analyse croisée de probabilités d'apparition des séquences sèches $\geq 10$ jours, 30 jours après le semis et pendant la floraison du maïs a permis de situer la période favorable du semis du maïs à partir de la troisième décade de Mai pour la zone soudanienne et de la première décade de Juin pour la zone soudanosahélienne. Ces résultats confirment les dates de recommandations sur les dates de semis du maïs au Burkina Faso (Sanou, 1989). La connaissance de la date favorable au semis permet de réduire les risques de ressemis ou d'occurrence de stress hydrique dommageable à la culture en phase végétative. En effet, les travaux de Pindard (2000) ont montré que même si le maïs peut tolérer un stress hydrique en période de montaison et jusqu'à la sortie des premières soies, des épisodes secs constituent néanmoins une contrainte pour l'installation et le bon développement du maïs.

Les résultats de l'analyse sur l'apparition des séquences sèches $>5$, et 7 jours à la floraison du maïs de 90 jours dans la zone d'étude, ont révélé que les probabilités d'apparition de séquences sèches $>5$ jours sont encore élevées pour les semis du mois de Mai sauf à Bobo. Celles > 7 jours bien que faibles pour la majorité des stations sont persistantes quelque soit la date de semis au mois de Mai et quelle que soit la zone agro écologique. Les probabilités d'apparition de séquences sèches $>10$ jours sont faibles voire nulles. Toutefois, leur occurrence quoique moins dommageable à la culture du maïs au stade de développement végétatif, a des impacts négatifs sur le rendement grain du maïs au stade de floraison/formation du grain. 
En effet, les travaux de Robelin (1963) ont montré que la période de très grande sensibilité du maïs commence 20 à 30 jours avant la floraison femelle et s'achève 10 à 15 jours après. Un manque d'eau à ce stade entraine une baisse de rendement de plus de 60\%. Au Togo, Poss et al. (1998) ont démontré que si les besoins en eau à la floraison du maiis sont satisfaits à moins de $60 \%$, les rendements deviennent très faibles, quelles que soient les conditions hydriques sur le reste du cycle.

De même, l'analyse des causes de la baisse des valeurs de l'indice I de satisfaction des besoins en eau du maïs a révélé que dans la zone soudano-sahélienne ce sont les déficits hydriques au cours de l'installation du maïs et surtout au moment de la floraison qui en sont principalement les causes. En revanche, en zone soudanienne, les baisses de rendement sont liées à des déficits hydriques mais aussi à quelques cas de surplus d'eau. En effet, Koné (1991) avait déjà constaté que la seule connaissance du régime pluviométrique ne suffit pas à expliquer le rendement du maïs pluvial, un rendement faible peut résulter aussi bien de conditions hydriques déficitaires qu'excédentaires.

L'apparition des séquences sèches et des déficits hydriques au cours du cycle du maïs en particulier à la floraison représente un risque majeur pour la culture du maïs au Burkina Faso en zone soudano-sahélienne. Tandis que les déficits hydriques et les surplus d'eau constituent les menaces de la culture du maïs en zone soudannienne.

Or, le changement climatique selon IPCC (2007) indique une recrudescence de phénomènes extrêmes tels que les fortes pluies et les inondations. Déjà l'Afrique de l'Ouest a enregistré au cours des années 2000 les pires inondations de ces 30 dernières années.

L'étude sur les durées de retour des pluies maximales cumulées en 3 jours consécutifs a montré que les pluies décennales dépassent $100 \mathrm{~mm}$ et peuvent atteindre plus de $130 \mathrm{~mm}$ dans la zone maïsicole. Une saison sur deux on enregistre au moins une pluie maximale de plus de $80 \mathrm{~mm}$. Ceci accentue le risque d'excès d'eau et d'engorgement des champs de maïs en zone soudanienne notamment. Ce qui sans doute explique que l'on ait observé à travers l'indice I, de cas fréquents d'excès d'eau pouvant être dommageables à la culture de maïs. L'engorgement du maïs consécutif à des pluies importantes constitue en zone soudanienne un risque majeur pour la culture du maïs. Toutefois, il faut souligner que le travail est conduit en considérant un stock d'eau de plus de $100 \mathrm{~mm}$ comme asphyxiant pour le système racinaire du maïs par manque d'oxygène. Toutefois, cette valeur pourra être calibrée et validée localement en fonction des caractéristiques hydro dynamiques des sols et de la sensibilité des variétés de maïs à l'excès d'eau.

\section{Conclusion}

Cette étude a permis de faire la synthèse des risques climatiques majeures pour la culture du maïs en zone soudanosahélienne et en zone soudanienne au Burkina Faso. Le maïs est une culture qui présente une grande sensibilité à la fois au déficit hydrique et à l'excès d'eau. En Afrique Sub saharienne, en particulier au Burkina Faso, il existe plusieurs risques climatiques associés à la variabilité temporelle des pluies, des paramètres de la saison, à la recrudescence de phénomènes tels que les fortes pluies. Tous ces facteurs, selon le profil de l'hivernage, peuvent constituer une contrainte climatique majeure pour la productivité du maïs.

Les analyses agroclimatiques conduites sur la période 1979-2008 dans la zone soudanienne et soudano-sahélienne ont montré que la variabilité des paramètres clés de la saison des pluies tels que les dates de démarrage, de fin et la longueur de la saison agricole ne constituent pas des risques majeurs pour la culture du maïs. La période d'installation des pluies et la longueur de la saison des pluies sont dans plus de $80 \%$ des années agricoles adéquates pour permettre à 
un maïs de 90 à 100 jours de boucler convenablement son cycle. En outre, en considérant les cumuls pluviométriques saisonniers dans la zone maïsicole, les besoins en eau totaux du maïs sont également satisfaits quelle que soit l'année et la zone. En revanche, l'analyse des séquences sèches au cours du cycle de développement du maïs d'une part, et l'analyse de l'indice I de satisfaction des besoins en eau d'autre part, ont permis d'identifier des contraintes majeures pour le maïs. Dans la zone soudanienne ce sont les excès d'eau, ou les excès d'eau conjugués à des déficits hydriques qui contribuent à réduire fortement la productivité du maïs. Tandis qu'en zone soudano-sahélienne, les risques agroclimatiques du maïs sont principalement dus à l'apparition de périodes de déficits hydriques. Ces résultats peuvent servir de base pour la mise en place de stratégies de prévention et de gestion de ces aléas climatiques.

\section{REMERCIEMENTS}

Ce document a été réalisé avec l'appui financier de l'Union Européenne (UE) dans le cadre de l'initiative «Global Climate Change Alliance » (GCCA). Les auteurs expriment leur gratitude à l'UE.

\section{REFERENCES}

Abdou A, Lebel T. 2008. Amani A. Signification et usage de l'indice pluviométrique au Sahel. Sécheresse, 19(4): 227-235.

Araya A, Stroosnijder L. 2011. Assessing drought risk and irrigation need in northern Ethiopia. Agri. For. Met., 151: 425-436.

Barry D. 2003. Contribution à la détermination des besoins en eau des principales cultures irriguées selon les zones agroclimatiques du Burkina Faso. Mémoire de fin d'études d'Ingénieur en Agrométéorologie, Centre Régional Agrhymet, Niamey, p. 56.
Bunasols. 2003. Etat de connaissance de la fertilité des sols du Burkina Faso. Documentation Technique $\mathrm{n}^{\circ} 1$, Ministère Agriculture et Elevage : Ouagadougou.

Camberlin P, Diop M. 2003. Application of daily rainfall principal component analysis to the assessment of the rainy season characteristics in Senegal. Clim. Res., 23: 159-169.

Chetaille A, Lagandré D. 2010. L'assurance indicielle, une réponse face aux risques climatiques. Grain de sel, 49: 20-21.

Christensen JH, Hewitson B, Busuioc A, Chen A, Gao X, Held I, Jones R, Kolli RK, Kwon WT, Laprise R, Rueda VM, Mearns L, Menéndez CG, Räisänen J, Rinke A, Sarr A, Whetton P. 2007. Regional Climate Projections. In Climate Change 2007: The Physical Science Basis. Contribution of Working Group I to the Fourth Assessment Report of the Intergovernmental Panel on Climate Change. Cambridge University Press: Cambridge, United Kingdom and New York.

Cochemé J, Franquin P. 1967. A Study of the Agroclimatology of the Semi Arid Area South of the Sahara in West Africa. FAO Unesco: Rome, Paris.

Dancette C. 1983. Besoins en eau du mil au Sénégal. Adaptation en zone semi-aride tropicale. Agro Trop., 38: 267-80.

DGPER. 2010. Analyse de la Compétitivité des Filières de Céréales Sèches au Burkina Faso. MAHRH Press: Ouagadougou.

Diop M. 1996. À propos de la durée de la saison des pluies au Sénégal. Sécheresse, 7(1): 7-15.

Diouf M, Nonguierma A, Abou A, Royer A, Somé B. 2000. Lutte contre la sécheresse au Sahel : acquis et perspective au Centre Régional Agrhymet. Sécheresse, 11(4): 257-266.

Djerboua A, Duband D, Bois P. 2004. Estimations des lois des précipitations extrêmes à partir des données journalières complètes. La Houille Blanche, 3: 65-74. 
Doorenbos J, Kassan AH. 1980. Réponse des rendements à l'eau. FAO, Bulletin d'irrigation et de Drainage 33, Rome.

Frere M, Popov GF.1987. Suivi agrométéorologique des cultures et prévision des rendements. Etude FAO Production végétale et protection des plantes 73,Rome.

Gachon P, Bokoye AI, Cotnoir A. 2007. Working Group II: Variability, extremes and climate change in the Sahel: from observation to modeling. Report on Canadian Contributions to the CIDACILSS Project (A030978-002) Climate Change Adaptation Capacity Support, Montréal (Québec).

IPCC. 2007. Climate Change 2007: Impacts, Adaptation and Vulnerability. Contribution of Working Group II to the Fourth Assessment Report of the Intergovernmental Panel on Climate Change, Parry ML, Canziani OF, Palutikof JP, van der Linden PJ, Hanson CE (Eds.), Cambridge University Press: Cambridge, UK.

Janicot S, Trzaska S, Poccard I. 2001. Summer Sahel-ENSO teleconnection and decadal time scale SST variations. Climate Dyn., 18: 303-320.

Kambire H, Abdel-Rahman G, Bacyé B, Dembele Y. 2010. Modeling of Maize Yields in the South-Sudanian Zone of Burkina Faso - West Africa. AmericanEurasian J. Agric. Environ. Sci., 7(2): 195-201.

Koné D. 1991. Caractérisation du risque climatique de la culture du maïs en zone centre de la Côte d'lvoire. In Soil Water Balance in the Sudano-Sahelian Zone, IAHS Publi : Niamey.

Le Barbé L, Lebel T, Tapsoba D. 2002. Rainfall Variability in West Africa during the Years 1950-1990. J. Clim., 15(2): 187-202.

Lebel T, Diedhiou A, Laurent H. 2003. Seasonal Cycle and Interannual Variability of the Sahelian Rainfall at
Hydrological Scales . J. Geo. Res., 108(8): 1-11.

Nicholson SE. 2001. Climatic and environmental change in Africa during the last two centuries. Clim. Res., 17: 123-144.

Omotosho JB, Balogun AA, Ogunjobi K. 2000. Predicting monthly and seasonal rainfall, onset and cessation of the rainy season in West Africa using only surface data. Int. J. Climatol., 20: 865-880.

Pindard A. 2000. La relation stress hydrique rendement du maïs en Bresse : quelle perspective de spatialisation? Utilisation d'un simulateur de culture (STICS). Mémoire d'Ingénieur d'Agronomie, ENSA, Dijon, p. 86.

Poss R, Saragoni H, Imbernon J. 1988. Bilan hydrique simulé du maïs au Togo méridional. Agr. Trop., 43(1): 18-29.

Robelin M. 1963. Contribution à l'étude du comportement du maïs grain vis-à-vis de la sécheresse. In Journée internationale de l'irrigation, AGPM, Agen.

Samba A. 1998. Les logiciels DHC de diagnostic hydrique des cultures, prévision des rendements du mil en zones soudano-sahéliennes de l'Afrique de l'Ouest. Sécheresse, 4(9): 281-288.

Sanou J. 1989. Etat de la Production du Maïs au Burkina Faso. Recommandations de la Recherche Agricole. INERA: Bobo Dioulasso.

Sarr B, Lona I. 2009. Les fortes pluies et les inondations enregistrées au Sahel au cours de l'hivernage 2007 : variabilité et / ou changement climatique. $14^{\text {ème }}$ Colloque International, SIFEE «Changement climatique et évaluation Environnementale» Outils et enjeux pour l'évaluation des impacts et l'élaboration des plans d'adaptation, Niamey.

Sarr B, Ndjendole S, Diouf O, Diouf M, RoyMacauley H. 1999. Suivi de l'état hydrique du sol et de la température du couvert de maïs au Sénégal. Sécheresse, 10(2): 129-135. 
Sivakumar MVK. 1988. Predicting rainy season potential from the onset of rains in southern sahelian and sudanian climatic zones of West Africa. Agr For. Meteorol., 42: 295-305.

Somé L, Sivakumar MVK. 1994. Analyse de la Longueur de la Saison Culturale en Fonction de la Date de Début des Pluies au Burkina Faso. INERA, ICRISAT: Ouagadougou.

Stern R, Dennett MN, Garbut DJ. 1981. The start of the rains in West Africa. Int. J. Climatol., 1: 59-68.

Stern R, Rijks D, Dale I, Knock J. 2006. INSTAT+ for Windows V 3.036. Statistical Services Center, University of Reading: Reading.

Stern RD, Cooper PMJ. 2011. Assessing climate risk and climate change using rainfall data : a case study from Zambia. Expl. Agric., 47(2): 241-266.

Tadross M, Suarez P, Lotsch A, Hachigonta S, Mdoka M, Unganai L, Lucio F, Kamdonyo D, Muchinda M. 2009. Growing-season rainfall and scenarios of future change in southeast Africa: implications for cultivating maize. Clim. Res., 40: 147-161.
Traoré SB, Reyniers FN, Vaksmann M, Kouressy M, Yattara K, Yoroté A, Sidibé A, Koné B. 2000. Adaptation à la sécheresse des écotypes locaux de sorgho du Mali. Sécheresse, 11(4): 227-237.

Traoré S, Agali A, Muller B, Kouressy M, Somé L, Sultan B, Oettli P, Siéné Laopé AC, Vintrou E, Sangaré S, Vaksmann M, Diop Mb, Bégué A, Dinghun M, Baron C. 2010. Characterizing and Modelling the Diversity of Cropping Situations under Climatic Constraints in West Africa. Accepted by Atmospheric Science Letters.

Watson, RT, Zinyowera MC, Moss RH. 1998. The Regional Impacts of Climatic Change: an Assessment of Vulnerability. A special report of IPCC Working group. Cambridge University Press : Cambridge, United Kingdom and New York.

Wey J. 1998. Etude de la variabilité du rendement du maïs au Burkina Faso. Thèse de doctorat, INPL, Nancy, p.200. 